\title{
Estrategia para mejorar la difusión de los resultados de investigación con la Web 2.0
}

\author{
Por Daniel Torres-Salinas y Emilio Delgado-López-Cózar
}

\begin{abstract}
Resumen: La comunicación científica se está viendo enriquecida por la utilización de nuevos modos de almacenamiento, publicación y difusión de los resultados. Entre ellos se encuentran los servicios de la Web 2.0, que aún son desconocidos en gran medida por los investigadores. En este contexto se indica cómo podemos emplear estratégicamente estos servicios para difundir y dar mayor visibilidad a las publicaciones científicas. Para ello se presenta una serie de aplicaciones de interés para la comunicación científica (Blogger, Twitter, Facebook, Slideshare y E-Lis) y se explica la función que pueden desempeñar para comunicar resultados científicos. A continuación se muestra cómo deben interrelacionarse lógicamente en lo que se ha denominado ciclo estratégico de difusión. Para concluir se recopila un conjunto de indicadores webmétricos clasificados en tres grupos (influencia social, utilización y reconocimiento) para conocer el éxito de los trabajos en la Web 2.0 y de la estrategia de difusión.
\end{abstract}

Palabras clave: Web 2.0, Ciencia 2.0, Publicación científica, Comunicación científica.

Title: Strategies to improve the dissemination of research results with the Web 2.0

Abstract: Scientific communication is being enriched by the introduction of new ways of storage, publication and dissemination of the results. These include the services of the Web 2.0

which are still largely unknown to researchers. In this context the objective of this paper is to illustrate how we can strategically use Web Services 2.0 to disseminate and give greater visibility to scientific publications. To this end we present a series of services of interest to scientific communication (Blogger, Twitter, Facebook, Slideshare y E-Lis) and explain the role they can play in communicating scientific results. It also shows how these services should be logically interrelated, which we call the strategic dissemination cycle. In the last section, the authors collected a set of webmetric indicators classified into three groups (social influence, use, recognition) to evaluate the success of the dissemination strategy and the visibility of a work in the Web 2.0.

Keywords: Web 2.0, Science 2.0, Scientific publication, Scientific communication.

Torres-Salinas, Daniel; Delgado-López-Cózar, Emilio. "Estrategia para mejorar la difusión de los resultados de investigación con la Web 2.0”. El profesional de la información, 2009, septiembre-octubre, v. 18, n. 5, pp. 534-539.

DOI: $10.3145 /$ epi.2009.sep.07

\section{Introducción}

EN LOS ÚLTIMOS AÑOS la comunicación científica se está viendo enriquecida por la utilización de nuevos modos de almacenamiento, publicación y difusión de resultados.

Nos encontramos en un momento de fragmentación de la comunicación científica (Brown;
Boulderstone, 2008) donde otros modos de transmitir los resultados de investigación empiezan a participar en el espacio reservado anteriormente a las revistas. Bohlin (2004), que habla de regímenes de comunicación, señala al menos tres tendencias bien consolidadas: repositorios, autoarchivo y revistas científicas. A éstas tendríamos que añadir recientemente las platafor- mas de edición y publicación nacidas en torno a la Web 2.0 o Web social.

Todos estos medios, especialmente los relacionados con el mundo 2.0, han traído un gran número de aplicaciones en su mayoría desconocidas por los investigadores, más apegados a las prácticas de publicación tradicionales. Es evidente que la comunidad científica aún 


\section{"La comunicación científica se está viendo enriquecida por los nuevos modos de almacenamiento, publicación y difusión de resultados"}

no tiene conciencia de su utilidad y hay enormes reticencias por la falta de reconocimiento que tengan sus resultados publicados en estos canales (Waldrop, 2008).

Sin embargo todo investigador debería tener claro que uno de los propósitos esenciales de la ciencia es la publicación de resultados ya que, como propugna Ziman (1998), la ciencia es ante todo un sistema de comunicación y estos canales son una oportunidad de fortalecer y optimizar dicho sistema. Según este autor la ciencia actual se caracteriza también por redes de trabajos donde la información se transmite entre los nodos de las mismas. La Web social no es otra cosa que un entramado digital de nodos que proporciona una cobertura tecnológica al concepto de Ziman. Los nuevos canales 2.0 pueden ser por tanto un escenario perfecto donde difundir las publicaciones.

El propósito de este trabajo es ilustrar sobre los múltiples usos y aprovechamientos que se pueden dar a las herramientas 2.0, en interacción con los repositorios, para difundir y comunicar resultados científicos, independientemente de que éstos sean inéditos o publicados, para alcanzar una mayor visibilidad y difusión.

En definitiva presentamos una estrategia para una transmisión más efectiva de los resultados científicos. Para ello tratamos en primer lugar de las aplicaciones generalistas 2.0 que podemos emplear; en segundo lugar cómo debemos interrelacionarlas y por último cómo podemos monitorizar métricamente esta estrategia para conocer si estamos obteniendo éxito.

\section{Servicios 2.0 útiles para la publicación científica}

Del numeroso conjunto de servicios que se ofertan en la Web 2.0 hemos seleccionado cuatro, además de los repositorios en acceso abierto, todos ellos de carácter gratuito. La selección se ha realizado en función del número de usuarios, es decir del tamaño de la comunidad y por tanto de su audiencia potencial; además, casi todos ellos actúan como estándares en el mundo 2.0 y en última instancia todos ofrecen indicadores que nos permitirán monitorizar cuantitativamente nuestra estrategia. Estas aplicaciones son las siguientes:
- Blogger: servicio de alojamiento de blogs que hace posible la publicación periódica y secuencial de entradas (posts) con elementos textuales y multimedia.

- Twitter: servicio que facilita a sus usuarios el lanzamiento de mensajes cortos de carácter textual (tweets) que no superen los 140 caracteres, por lo que se conocen también como microblogs.

- Facebook: red social donde los usuarios pueden crear una cuen-

\section{"Las herramientas 2.0 permiten difundir y comunicar resultados científicos para alcanzar una mayor visibilidad"}

\begin{tabular}{|c|c|}
\hline \multicolumn{2}{|c|}{ Servicios de alojamientos de blogs } \\
\hline Blogger & https://www.blogger.com \\
\hline Wordpress & http://es.wordpress.com \\
\hline La Coctelera & http://www.lacoctelera.com \\
\hline Bitácoras.com & http://bitacoras.com \\
\hline \multicolumn{2}{|c|}{ Servicios de microblogging } \\
\hline Twitter & http://twitter.com \\
\hline Jaiku & http://www.jaiku.com \\
\hline Khaces & http://www.khaces.com \\
\hline \multicolumn{2}{|c|}{ Servicios de redes sociales } \\
\hline Facebook & http://www.facebook.com \\
\hline Tuenti & http://www.tuenti.com \\
\hline Hi5 & http://hi5.com \\
\hline Orkut & http://www.orkut.com \\
\hline \multicolumn{2}{|c|}{ Servicios de alojamiento de presentaciones } \\
\hline Slideshare & http://www.slideshare.net/ \\
\hline Zoho Show & http://show.zoho.com \\
\hline Google docs & http://docs.google.com \\
\hline \multicolumn{2}{|c|}{ Repositorios temáticos } \\
\hline E-Lis [documentación] & http://eprints.rclis.org/ \\
\hline arXiv.org [física, matemáticas] & http://arxiv.org \\
\hline RePEc [economía] & http://repec.org \\
\hline PubMed Central [ciencias de la salud] & http://www.pubmedcentral.nih.gov \\
\hline
\end{tabular}

Tabla 1. Breve directorio de servicios 2.0 y repositorios en acceso abierto 
ta con contactos y compartir todo tipo de materiales (vídeo, texto, etc.). Cuenta con 200 millones de usuarios y una amplia penetración en el mundo universitario.

- Slideshare: plataforma de alojamiento de presentaciones que pueden ser visualizadas mediante un reproductor e integradas en otros sitios web.

- Repositorios: archivos digitales donde las comunidades científicas suben materiales poniéndolos a libre disposición. En el campo de la documentación contamos con $E$ Lis, que utilizaremos como ejemplo.

Sin embargo aunque éstas son las aplicaciones que proponemos, hay que tener claro que existen otras similares (en la tabla 1 se presentan sólo algunos ejemplos) por lo que la selección de una u otra dependerá en última instancia de la comunidad a la que nos dirijamos y habrá que optar por aquellas donde se produzcan las interacciones sociales de nuestra disciplina. Así por ejemplo, es evidente que en el caso de los repositorios debemos escoger aquel que más se ajuste al tema de nuestra especialidad. Una primera forma de comprobar la audiencia potencial de los servicios de redes sociales es la búsqueda automática que suelen realizar estas aplicaciones de los contactos que tenemos en nuestras cuentas de correo para ver cuántos de ellos están suscritos al servicio. Se recomienda asimismo utilizar en todas las aplicaciones el mismo nombre de usuario y que éste sea fácilmente identificable con nuestra firma científica (por ejemplo si nuestra firma científica es $A$. López-López utilizar como usuario Alopezlopez).

\section{Función de las aplicaciones 2.0 y ciclo estratégico de difusión}

Cada servicio 2.0 tiene una función específica en el proceso de difusión de los resultados de inves-

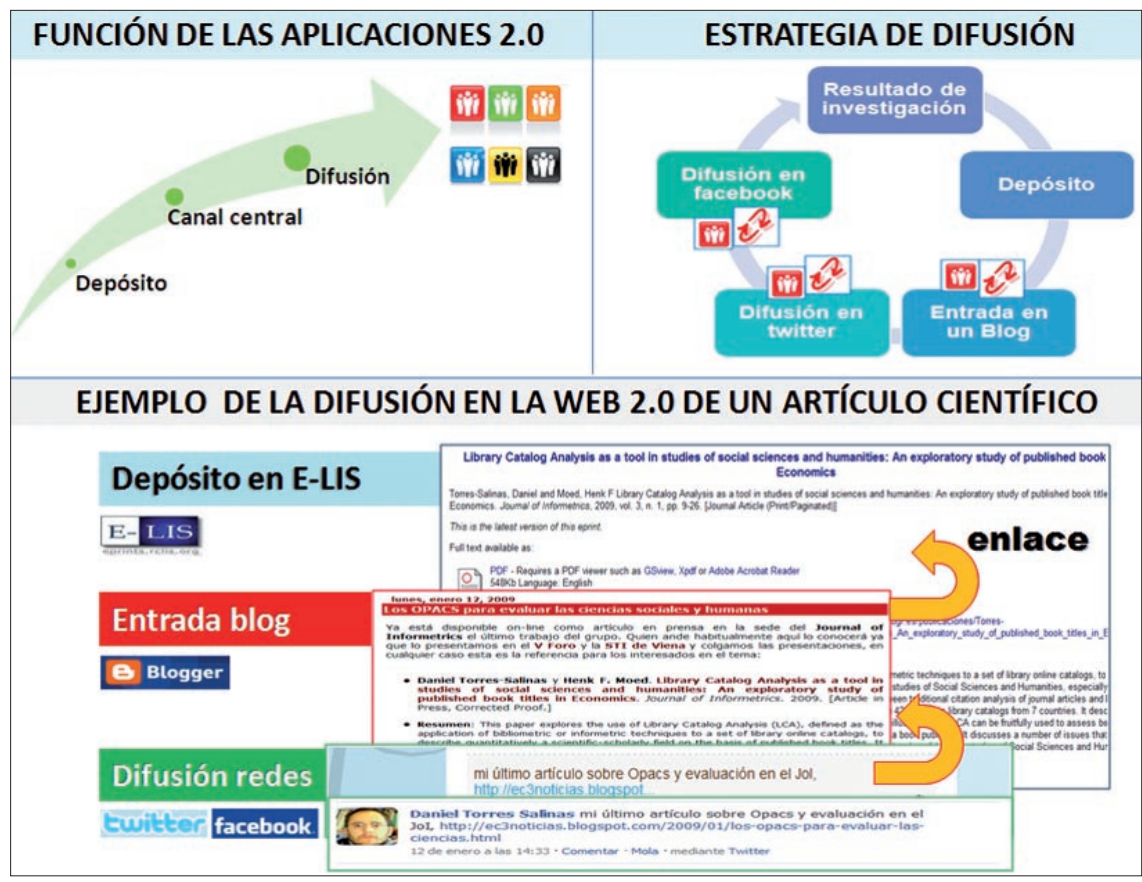

Figura 1. Función de las aplicaciones 2.0 en la difusión de resultados y ciclo estratégico de difusión

tigación. Podemos distinguir al menos tres usos (figura 1):

- Aplicaciones de depósito. Las vamos a aprovechar en primer lugar para el depósito y almacenamiento abierto de nuestras publicaciones. Es el caso de los repositorios como $E$-Lis, que emplearemos para depositar nuestros artículos, y Slideshare donde fundamentalmente colgaremos el material en pdf o ppt de las aportaciones realizadas en congresos o cursos.

- Canal central. Será el blog el que juegue el papel de canal central en el proceso de difusión ya que nos servirá para conectar las aplicaciones de depósito con los servicios de redes sociales. Será nuestra imagen en internet al ser recuperable desde cualquier buscador y desde él podemos anunciar todas nuestras actividades o resultados. Es además un medio que empieza a ser aceptado entre la comunidad científica (Torres-Salinas; Cabezas-Clavijo, 2008).

- Canales de difusión. Aunque todas las aplicaciones en última instancia sirven para dar difusión y visibilidad, las redes sociales recomendadas (Twitter y Facebook) son idóneas para el anuncio inicial de las publicaciones y para comenzar a introducirlas en el circuito científico y profesional. Si mantenemos una ecología de contactos adecuada, seleccionando únicamente contactos profesionales presumiblemente interesados en nuestras líneas de investigación, ellos serán los primeros en conocer nuestros resultados iniciando el proceso de difusión entre los nodos y jugando un rol similar al del envío de las tradicionales separatas por correo electrónico.

Una vez determinada la función de cada uno de estos servicios hay que tener en cuenta la interrelación entre los mismos y el momento en el que hay que usar cada uno de ellos. Es lo que denominamos el ciclo estratégico de difusión (figura $1)$, que tendría diferentes etapas.

El ciclo debe comenzar con el depósito de nuestro trabajo en las aplicaciones recomendadas. Hay que señalar que cuando se trate de artículos aceptados para ser publicados en una revista científica el autor debe estar atento a las políticas de autoarchivo de la misma ya que, dependiendo del editor, la 
posibilidad de poner en libre acceso el texto podría estar sometida a diferentes restricciones. Buena parte de las grandes editoriales científicas (Kluwer, Springer o Elsevier) no impiden el depósito de preprint y postprint aunque debe realizarse bajo ciertas condiciones. Por ejemplo, esta revista El profesional de la información permite el autoarchivo inmediato del artículo postprint siempre que se cite el url de su web. Antes de realizar el depósito, recomendamos consultar el directorio Sherpa-Romeo donde se detallan las políticas de copyright y autoarchivo de las editoriales científicas.

\section{http://www.sherpa.ac.uk/romeo}

Después de depositar el trabajo se redactará una entrada en el blog con el fin de informar a la comunidad de la libre disposición del mismo. Esta entrada debe tener un título descriptivo y el contenido debe recoger como mínimo la descripción bibliográfica completa (resumen incluido) y, si se desea y se cuenta con tiempo para ello, algunos comentarios del autor en un tono más divulgativo, explicando aspectos destacados de los resultados. No debemos olvidar incluir un enlace directo al documento en su lugar de depósito. Además si se trata de presentaciones depositadas en Slideshare podemos incrustar la presentación en la entrada gracias al código fuente que nos ofrece esta aplicación.

\section{"Los trabajos divulgados por cauces tradicionales también tienen cabida en el puzzle de las tecnologías 2.0"}

Por último redactaremos un mensaje corto en Twitter y Facebook para anunciar la publicación a nuestros contactos ofreciendo en esta ocasión un enlace que remita a los lectores directamente a la entrada del blog.

Con el ciclo propuesto habremos conseguido que todos los servicios queden lógicamente conectados entre sí, con una función específica para cada uno de ellos.

\section{La medición de la visibilidad}

Uno de los objetivos principales de la difusión de resultados mediante este tipo de aplicaciones es conseguir una mayor visibilidad en internet que no tiene que traducirse necesariamente en un mayor impacto científico (medido mediante la citación) pero sí en un aumento directo en la utilización de los materiales. Para comprobar si nuestra estrategia funciona, tanto las aplicaciones 2.0 como la mayor parte de los repositorios nos permiten comprobar el interés despertado por nuestros resultados e incluso conocer por quién, dónde y cuándo es consultado el material. Podemos clasificar los indicadores ofrecidos por estos servicios en tres grupos (figura 2):

\section{Indicadores de influencia social:}

- Número de nodos: los contactos profesionales que hemos agregado en nuestras cuentas de las diferentes redes sociales como Twitter y Facebook. También podemos considerar como nodos el número de usuarios suscritos al feed de nuestro blog desde agregadores como Bloglines o Google Reader.

- Número de comentarios y número de réplicas: son las opiniones a modo de comentarios dejadas por otros usuarios en las diferentes entradas realizadas en las aplicaciones. Se pueden medir en el blog pero también en Facebook y en Slideshare. En Twitter podemos considerar como réplica la opción reply to y en Facebook la opción like.

\section{Indicadores de utilización:}

- Visitas: se pueden contabilizar mediante los múltiples sistemas de contadores y estadísticas existentes, que ofrecen además otro tipo de informaciones de gran utilidad (origen, intermediarios, comportamiento, etc.) (Ortega; Aguillo, 2009). Utilizaremos estos sistemas, por ejemplo Google Analytics, para monitorizar la utilización del blog y sus diferentes entradas.

- Número de reproducciones y número de reutilizaciones de las presentaciones: ambos indicadores son ofrecidos por Slideshare y nos permiten, en primer lugar, saber el número de usuarios que han reproducido nuestra presentación y, en segundo, aquellas presentaciones que han sido incluidas en otros sitios webs siendo reutilizadas y difundidas por otros usuarios.

- Número de descargas y número de lecturas del resumen: la cantidad de usuarios que han descargado nuestros trabajos es una información facilitada tanto por Slideshare como por los repositorios, como es el caso de E-Lis. Además, este último nos indica desde qué país se ha realizado. Junto con las descargas, E-Lis nos ofrece también el número de lecturas o visualizaciones del abstract.

\section{Indicadores de reconocimiento:}

- Número de enlaces: tradicionalmente los links se han considerado el equivalente de la citas en la Web (sitation). Se pueden considerar los links recibidos por la entrada de nuestro blog en la que hemos anunciado la publicación, y pueden ser recuperados mediante servicios como Technorati o Google Blogs. También se deberían tener en cuenta los enlaces que reciben los documentos en sus lugares de depósito.

- Número de citas recibidas en Google Scholar: otra de las ventajas de la mayor parte de los repositorios, como ocurre en E-Lis, es 


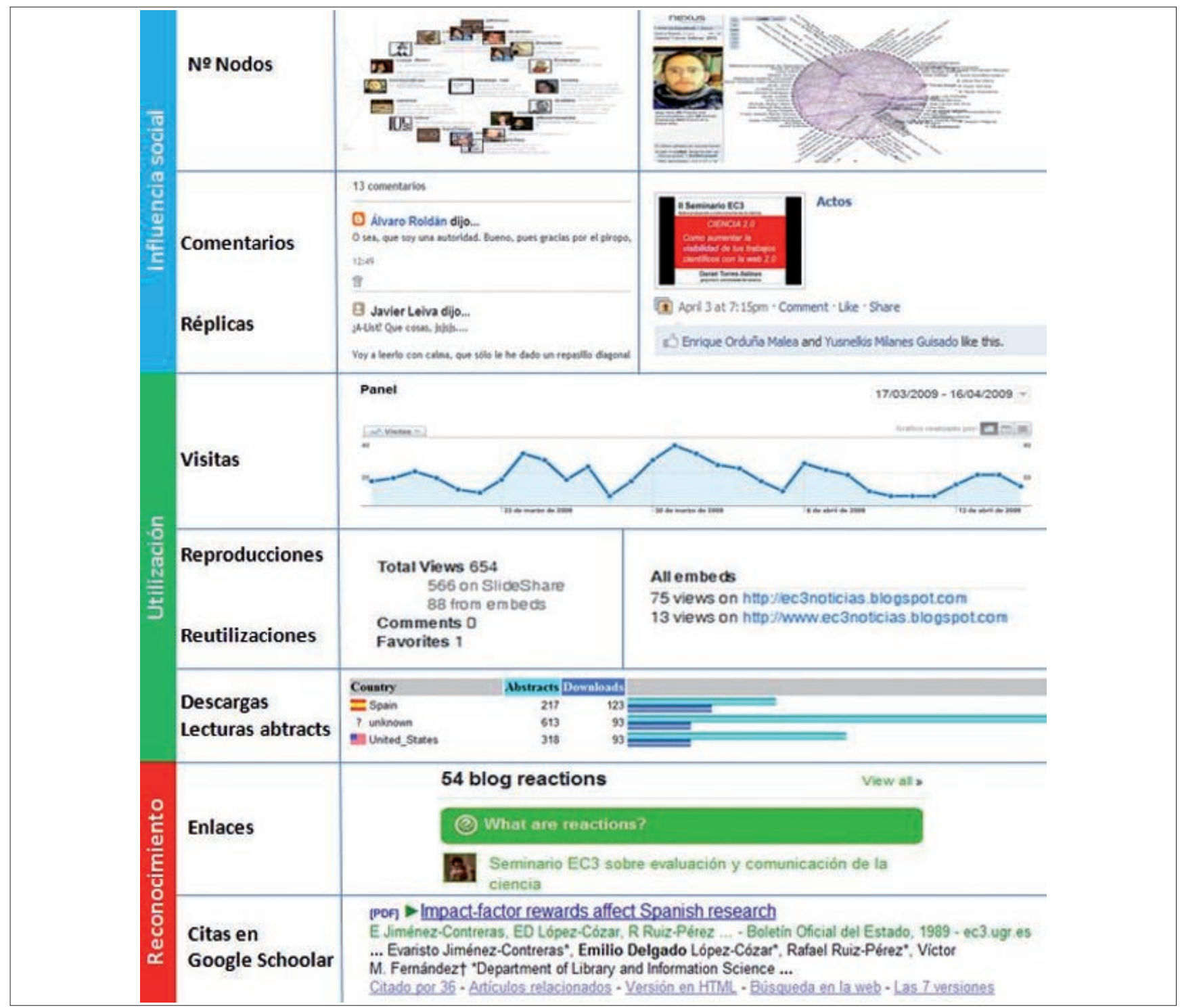

Figura 2. Ejemplo de algunos de los indicadores para monitorizar la visibilidad de nuestros trabajos en la Web 2.0

que su contenido es indizado y recuperable por otros servicios como Google Scholar permitiéndonos rescatar las citas recibidas.

\section{"La publicación científica no debe acabar con la aceptación de un manuscrito por parte de una revista"}

Como ejemplo real de estos indicadores podemos ilustrar cómo los autores de este trabajo en una aportación realizada en una mesa redonda titulada Qué y cómo se edita una revista científica 2.0 ante un público presencial de 50 personas alcanzaron los siguientes resultados en la Web 2.0: la presentación ha sido reproducida en 809 ocasiones, descargada en 31 , la entrada tiene una media de 29 visitas mensuales, recibió 2 comentarios, 1 enlace y la información llegó directamente a sus 30 contactos en Twitter y los 103 de Facebook. Gracias a la estrategia de difusión hemos pasado claramente a una audiencia más amplia y variada de la original conseguida con la suma de las microaudiencias de las diferentes aplicaciones. Analizando los indicadores mostrados, estas aplicaciones nos pueden ayudar a conocer cuáles son aquellos de nuestros trabajos que más respaldo e interés despiertan, lo que en cierta medida nos puede orientar temáticamente. En definitiva, estas aplicaciones están generando una serie de nuevos indicadores webmétricos que podíamos denominar de segunda generación y la suma de estas medidas estarían muy cerca de lo que Cronin (2005) denomina Presence Density o Scholarly Salience.

\section{Consideraciones finales}

Cuando se habla de modelos de comunicación se tiende a tomar una postura determinista y dicotómica en la que se cuenta con la inadecuación de la postura contraria o con la elección al menos por parte del investigador de una ellas (acceso abierto/acceso por pago, o publicación 2.0/revistas tradicionales). Sin embargo, tal como hemos expuesto, 
nos encontramos en un momento en el que hay diferentes regímenes de comunicación y ninguno de ellos acabará monopolizando la publicación científica. El escenario al que nos enfrentamos es el de una coexistencia pacífica entre distintos modelos no necesariamente excluyentes.

Con la estrategia propuesta se propugna que los trabajos divulgados por cauces tradicionales puedan ser reaprovechados en otros circuitos ya que también tienen cabida en el puzzle de las tecnologías 2.0. De esta forma, un trabajo científico difundido en medios formales llegará a su comunidad natural, siendo reconocido su valor mediante la citación; pero además, difundiéndolo con las herramientas de la Web social cobra una nueva vida con una mayor audiencia potencial y un público más diversificado, como pueden ser los colectivos profesionales.
Por tanto creemos que la publicación científica no debe acabar con la aceptación de un manuscrito por parte de una revista y que es responsabilidad del científico, individual y social, dar la mayor publicidad posible a su trabajo.

\section{Bibliografía}

Ortega, José-Luis; Aguillo, Isidro F. "Minería del uso de webs". El profesional de la información, 2009, v. 18, n. 1, pp. 20-26.

Bohlin, Ingemar. "Communication regimes in competition: the current transition in scholarly communication seen through the lens of the sociology of technology". Social studies of science, 2004 , v. 34 , n. 3 , pp. 365-391.

Brown, David J.; Boulderstone, Richard. The impact of electronic publishing. München: KG Saur, 2008. ISBN 978-3598115158.

Cronin, Blaise. The hand of science: academic writing and its rewards. Lanham, Toronto, Oxford: Scarecrow Press, 2005, ISBN 9780810852822 .

Torres-Salinas, Daniel; Cabezas-Clavijo, Álvaro. "Los blogs como nuevo medio de comunicación científica". En: III Encuentro ibérico de docentes e investigadores en información y documentación, 2008, pp. 773-781.

Ziman, John. Real science: what it is, and what it means. Cambridge: Cambridge University Press, 1998, ISBN 0521471710.

Waldrop, M. Mitchell. "Science 2.0 - Is open access science the future?". Scientific American, 2008 , v. 298 , n. 5 , pp. $68-73$.

\section{Daniel Torres-Salinas}

Grupo Evaluación de la Ciencia y la Comunicación Científica (EC3), Centro de Investigación Médica Aplicada, Universidad de Navarra, Av. Pío XII, 31008 Pamplona (España).

torressalinas@gmail.com

\section{Emilio Delgado-López-Cózar}

Grupo Evaluación de la Ciencia y la Comunicación Científica (EC3), Departamento de Biblioteconomía y Documentación, Universidad de Granada, Colegio Máximo, Campus Cartuja, 18001 Granada (España). edelgado@ugr.es

\section{Don't Federate - Integrate EBSCOhost' Integrated Search}

Hasta la fecha, los servicios de búsquedas federadas han sufrido cuatro problemas principales:

- Pocas funciones y herramientas

- Lentitud en las búsquedas

- Poco servicio al cliente

- Altos costes y cuotas de mantenimiento

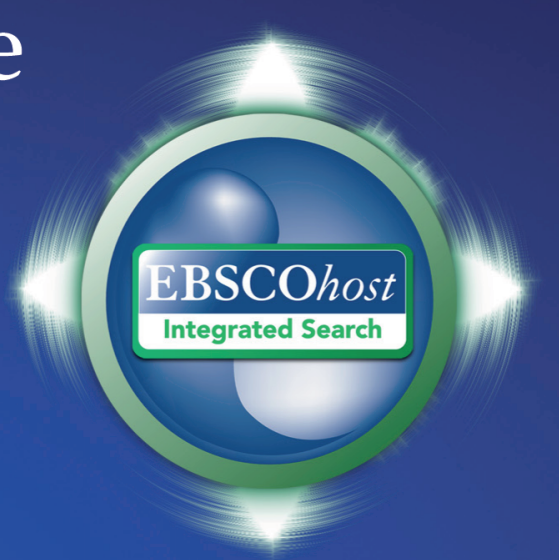

EBSCOhost Integrated Search" lleva las búsquedas federadas a un nivel más alto, ofreciendo excelentes soluciones a estos problemas:

1 Avanzadas herramientas y funciones

Simultáneamente búsqueda de bases de datos de EBSCO y otros recursos electrónicos usando la plataforma EBSCOhost y sus herramientas.

2 Respuesta rápida a las búsquedas realizadas Los resultados de las búsquedas son presentados por niveles, resultados de niveles más altos son presentados rápidamente, mientras que los más lentos o menos relevantes al tema, van buscando mientras se muestran los primeros resultados.
3 Servicio al Cliente de primer nivel.

Soporte técnico en el país, así como soporte en línea los 7 días de la semana las 24 horas.

4 Grandes ahorros en precio

EBSCO no cobrará los conectores a las bases de datos que estén a través de EBSCOhost, lo que ahorrará considerablemente los precios por conectores creados, al momento de solicitar el presupuesto.

Para más información visite www.ebscohost.com/integrate, o escriba un correo a request@ebscohost.com 